\title{
BOUNDED ANALYTIC FUNCTIONS ON TWO SHEETED DISCS
}

\author{
MIKIHIRO HAYASHI, MITSURU NAKAI, AND SHIGEO SEGAWA
}

To Professor Tatsuo Fuji'i'e on his sixtieth birthday

\begin{abstract}
Results of qualitative nature of both positive and negative directions on the point separation by bounded analytic functions of smooth subregions of two sheeted discs are given when two sheeted discs themselves are not separated by bounded analytic functions. We are, in particular, concerned about roles of branch points in two sheeted discs played in the point separation by bounded analytic functions.
\end{abstract}

Consider two sheeted unlimited covering surface $\left(\Delta^{\sim}, \Delta, \pi\right)$ of the open unit disc $\Delta$ with its covering map $\pi$ whose branch points are those over the sequence $\left\{z_{n}\right\}$ of points $z_{n}$ in $\Delta$. We are interested in the situation when $H^{\infty}\left(\Delta^{\sim}\right)$ does not separate the points in the fiber $\pi^{-1}(z)$ over any point $z$ in $\Delta$, i.e.,

$$
H^{\infty}\left(\Delta^{\sim}\right)=H^{\infty}(\Delta) \circ \pi,
$$

where we denote by $H^{\infty}(R)$ the class of bounded holomorphic functions on a Riemann surface $R$. We know (cf. 1.3) that (1) holds if and only if

$$
\sum_{n \geq 1}\left(1-\left|z_{n}\right|\right)=\infty \text {. }
$$

Concerning the result mentioned above we usually understand that two sheets in $\Delta^{\sim}$ are so tightly put together by the presence of the branch points satisfying (2) that $\Delta^{\sim}$ behaves like one sheeted in the sense of (1) from the view point of being the domains of definition of bounded holomorphic functions. It is this point that we wish to analyze in more detail in this paper: we ask whether the existence of the branch points with the condition (2) is really essential for the validity of (1).

In order to make the problem more precise we will take the closed disc $\Delta_{k}$ with its center $z_{k}$ contained in $\Delta$ for each $k \geq 1$ in such a fashion that $\left\{\Delta_{k}\right.$ : $k \geq 1\}$ are disjoint by pairs; then consider the subregion

$$
D=\Delta \backslash \bigcup_{k \geq 1} \Delta_{k},
$$

Received by the editors April 26, 1990 and, in revised form, August 8, 1990.

1980 Mathematics Subject Classification (1985 Revision). Primary 30F20; Secondary 30D50, 30D55, 46J10.

This work was partly supported by Grant-in-Aid for Scientific Research, No. 01540120, Japanese Ministry of Education, Science, and Culture. 
of $\Delta$ which gives rise to the two sheeted smooth covering surface $\left(D^{\sim}, D, \pi\right)$ naturally associated with $\left(\Delta^{\sim}, \Delta, \pi\right)$. If the existence of branch points in $\Delta^{\sim}$ with (2) were really essential for the validity of (1), we would no longer be able to expect the validity of the relation

$$
H^{\infty}\left(D^{\sim}\right)=H^{\infty}(D) \circ \pi,
$$

since in $D^{\sim}$ all the branch points in $\Delta^{\sim}$ are removed. We can now exactly formulate our problem in this paper as follows: When do we have the situation (3)?

A simple minded guess would say that (3) is valid if we take $\left\{\Delta_{k}\right\}$ small enough and invalid if we choose $\left\{\Delta_{k}\right\}$ as large as possible. We will see that the former is certainly the case but the latter is not quite right unless the assertion is somewhat weakened. More precisely we will show the following four results in this paper.

(a) The relation (3) is always valid for any covering surface $\left(\Delta^{\sim}, \Delta, \pi\right)$ with (2) by choosing the sequence $\left\{\Delta_{k}\right\}$ of discs in $\Delta$ suitably and actually small enough.

(b) There exists a covering surface $\left(\Delta^{\sim}, \Delta, \pi\right)$ with (2) such that (3) is always valid no matter how we choose the sequence $\left\{\Delta_{k}\right\}$ of discs in $\Delta$.

(c) There exists a covering surface $\left(\Delta^{\sim}, \Delta, \pi\right)$ with (2) such that (3) is invalid by choosing the sequence $\left\{\Delta_{k}\right\}$ of discs in $\Delta$ suitably.

(d) If we allow ellipses in place of discs as $\Delta_{k}$ one of whose foci is $z_{k}$ and whose major axes tend to zero, then (3) is invalid for any covering surface $\left(\Delta^{\sim}, \Delta, \pi\right)$ by choosing the sequence $\left\{\Delta_{k}\right\}$ of ellipses in $\Delta$ suitably.

After preliminaries covered in $\S 1$ the result (a) above, which is the main result of this paper, will be given and proven as Theorem 1 in $\S 2$ titled nonseparating discs. More than stated in (b) ((c), resp.) above we will show as Theorem 2 $(3$, resp.) in $\S 3(4$, resp.) with the subtitle rigid covering surfaces (nonrigid covering surfaces, resp.) that the covering surface $\left(\Delta^{\sim}, \Delta, \pi\right)$ in the statement (b) ((c), resp.) will be obtained by reforming an arbitrarily given covering surface with (2) by adding two new branch points (one new branch point, resp.) each arbitrarily in a sufficiently small neighborhood of each original branch point. In the last section, titled separating ellipses, the above result (d) will be given and proven as Theorem 4 .

We discussed the similar problem for the case of covering surfaces of the open unit punctured disc $\Delta_{0}=\Delta \backslash\{0\}$ the sequence of projections of whose branch points converges to 0 in our former papers [4 and 5] in which the results obtained were of quantitative character. Our results in this paper are entirely qualitative which we intentionally aimed for. But we feel it also important and interesting to obtain quantitative versions of our present results. These will be discussed elsewhere. We also remark that the method used in this paper can be reformed to cover the case of $\Delta_{0}$ instead of $\Delta$ and many of the results in this paper moreover generalize to more general Riemann surfaces including of course $\Delta$ and $\Delta_{0}$.

\section{Preliminaries}

1.1. We denote by $H^{\infty}(R)$ the Banach algebra of bounded holomorphic functions $f$ on a Riemann surface $R$ equipped with the supremum norm

$$
\|f\|=\|f\|_{R}=\sup \{|f(p)|: p \in R\} .
$$


For any subset $X$ of $R$ and nonempty subset $Y$ of $H^{\infty}(R)$ we say that $Y$ separates the points in $X$ if $X$ contains at least two points and there exists an $f$ in $Y$ for any pair of distinct points $p$ and $q$ in $X$ such that $f(p) \neq f(q)$. Hence $Y$ does not separate the points in $X$ if $X$ is a singleton or empty.

Consider a two sheeted unlimited covering Riemann surface $\left(R^{\sim}, R, \pi\right)$ of a Riemann surface $R$ with its holomorphic covering map $\pi$. It can happen that two sheets of $R^{\sim}$ are so tightly connected that $H^{\infty}\left(R^{\sim}\right)$ does not separate the points of any fiber $\pi^{-1}(p)$ over any point $p$ in $R$, i.e.,

$$
H^{\infty}\left(R^{\sim}\right)=H^{\infty}(R) \circ \pi
$$

where $H^{\infty}(R) \circ \pi=\left\{f \circ \pi: f \in H^{\infty}(R)\right\}$. In 1949 this phenomenon was first pointed out by P. J. Myrberg [6] when $R^{\sim}$ is the two sheeted unlimited covering surface of the open unit punctured disc such that the sequence of projections of its branch points converges to zero, which, though simple, had a great impact upon later developments concerning various theories related to bounded analytic functions (cf. e.g. [1, 3, 8], etc.). For this reason we say that the Myrberg type phenomenon occurs for $\left(R^{\sim}, R, \pi\right)$ if (1.1) is valid.

There is a theorem of Forelli [2] (cf. also Royden [7]) assuring as a special case that $(1.1)$ holds if $H^{\infty}\left(R^{\sim}\right)$ does not separate the points in the unramified fiber $\pi^{-1}(p)$ over at least one point $p$ in $R$ provided that $R$ satisfies a certain condition that Forelli calls divisibility which is satisfied by e.g. plane regions $R$. We will use it in the following form:

Fact 1. The Myrberg type phenomenon occurs for two sheeted unlimited covering surface $\left(S^{\sim}, S, \pi\right)$ of a bounded plane region $S$ if and only if $H^{\infty}\left(S^{\sim}\right)$ does not separate the points in the unramified fiber $\pi^{-1}(a)$ over at least one point a in $S$.

Since a very simple proof can be provided to such a restricted statement as above, we will give it in the next section for the sake of convenience and completeness.

1.2. Only the sufficiency part requires a nontrivial proof. Suppose that $H^{\infty}\left(S^{\sim}\right)$ does not separate the points in the fiber $\pi^{-1}(a)=\left\{a_{1}, a_{2}\right\}$ over a point $a$ in $S$. Consider the function $I_{a}$ in $H^{\infty}(S)$ given by $I_{a}(z)=z-a$ on $S$ and the function $h$ in $H^{\infty}\left(S^{\sim}\right)$ given by $h=I_{a} \circ \pi$. Take a small disc $V$ about $a$ contained in $S$ such that $\pi^{-1}(V)$ does not contain any branch point of $S^{\sim}$ and denote by $V_{1}$ and $V_{2}$ two components of $\pi^{-1}(V)$ such that $a_{j} \in V_{j}$ $(j=1,2)$. Observe that $h$ can be used as local parameters on $V_{j}(j=1,2)$.

Choose an arbitrary function $f$ in $H^{\infty}\left(S^{\sim}\right)$ and expand it into Taylor series in $h$ on $V_{j}$ :

$$
f=\sum_{k \geq 0} c_{j k} h^{k} \quad(j=1,2) .
$$

Note that $c_{10}=f\left(a_{1}\right)=f\left(a_{2}\right)=c_{20}$. We further maintain that $c_{1 k}=c_{2 k}$ for every $k \geq 1$. Contrary to the assertion assume that there exists the smallest integer $m \geq 1$ such that $c_{1 m} \neq c_{2 m}$. We can set $c_{k}=c_{1 k}=c_{2 k} \quad(0 \leq k<m)$. Then consider the function $g=\left(f-\sum_{k<m} c_{k} h^{k}\right) / h^{m}$, which is seen to belong to $H^{\infty}\left(S^{\sim}\right)$. However, by virtue of $(1.2)$, we see that

$$
g\left(a_{1}\right)=c_{1 m} \neq c_{2 m}=g\left(a_{2}\right),
$$


which contradicts the fact that $H^{\infty}\left(S^{\sim}\right)$ does not separate the points in $\pi^{-1}(a)=\left\{a_{1}, a_{2}\right\}$. Hence we can set $c_{k}=c_{1 k}=c_{2 k} \quad(k \geq 0)$.

Consider an arbitrary $z$ in $V$ and set $\pi^{-1}(z)=\left\{z_{1}, z_{2}\right\}$. Since $h\left(z_{1}\right)=$ $h\left(z_{2}\right)$ and $f=\sum_{k \geq 0} c_{k} h^{k}$ both on $V_{1}$ and $V_{2}$, we see that $f\left(z_{1}\right)=f\left(z_{2}\right)$. This means that $F(z) \equiv 0$ on $V$ for the function $F$ in $H^{\infty}(S)$ given by

$$
F(z)=\left(f\left(z_{1}\right)-f\left(z_{2}\right)\right)^{2} \text {. }
$$

A fortiori $F \equiv 0$ on $S$ and therefore $f\left(z_{1}\right)=f\left(z_{2}\right)$ for every $z$ in $S$ with $\pi^{-1}(z)=\left\{z_{1}, z_{2}\right\}$, or equivalently, $H^{\infty}\left(S^{\sim}\right)=H^{\infty}(S) \circ \pi$, completing the proof of Fact 1 .

1.3. We denote by $\Delta(c, r)(\bar{\Delta}(c, r)$, resp.) the open (closed, resp.) disc in the complex plane $\mathbf{C}$ with center $c$ and radius $r>0$. We simply denote by $\Delta$, as before, the open unit disc $\Delta(0,1)$.

Consider a two sheeted unlimited covering Riemann surface $\Delta^{\sim}$, or more precisely $\left(\Delta^{\sim}, \Delta, \pi\right)$, of the base surface $\Delta$ with its holomorphic covering map $\pi$. Unless the contrary is explicitly stated we always understand throughout this paper that the covering surface $\left(\Delta^{\sim}, \Delta, \pi\right)$ in consideration is of this sort.

A covering surface $\left(\Delta^{\sim}, \Delta, \pi\right)$ is uniquely determined by the sequence $\left\{p_{n}\right\}$ of its branch points $p_{n}$ in $\Delta^{\sim}$ and $\left\{p_{n}\right\}$ is uniquely determined by the discrete sequence $\left\{z_{n}\right\}$ of points $z_{n}=\pi\left(p_{n}\right)$ in $\Delta$. Thus a covering surface $\left(\Delta^{\sim}, \Delta, \pi\right)$ determines and is determined by a discrete sequence $\left\{z_{n}\right\}$ of distinct points in $\Delta$ which will be referred to as the determining sequence of $\left(\Delta^{\sim}, \Delta, \pi\right)$.

The Myrberg type phenomenon had already been observed by Selberg [10] in 1936 much earlier than Myrberg in a different context (cf. e.g. $[9,11,13]$, etc.) which in particular contains the following:

Fact 2. The Myrberg type phenomenon occurs for a covering surface $\left(\Delta^{\sim}, \Delta, \pi\right)$ if and only if its determining sequence $\left\{z_{n}\right\}$ satisfies the condition

$$
\sum_{n \geq 1}\left(1-\left|z_{n}\right|\right)=\infty \text {. }
$$

The sufficiency proof of the above will be found in $\$ 2.4$ below among the proofs of more general assertions. In the present case it goes as follows. Suppose there exists an $f$ in $H^{\infty}\left(\Delta^{\sim}\right) \backslash H^{\infty}(\Delta) \circ \pi$. Then the function $g$ in $H^{\infty}(\Delta) \backslash\{0\}$ given by $g(z)=\left(f\left(z^{+}\right)-f\left(z^{-}\right)\right)^{2}$ with $\pi^{-1}(z)=\left\{z^{+}, z^{-}\right\}$for each $z$ in $\Delta$ vanishes on $\left\{z_{n}\right\}$ and (1.3) is invalid (cf. e.g. [12]).

Since the idea of the necessity proof of the above will be used in $\S \S 4$ and 5, it will be helpful to exhibit here the proof as a prototype. Thus suppose that (1.3) is invalid. Then the square root $\sqrt{B}$ of the Blaschke product $B$ given by

$$
B(z)=\prod_{n \geq 1} \frac{-\bar{z}_{n}}{\left|z_{n}\right|} \cdot \frac{z-z_{n}}{1-\bar{z}_{n} z},
$$

belongs to $H^{\infty}\left(\Delta^{\sim}\right) \backslash H^{\infty}(\Delta) \circ \pi$ where we understand $-\bar{z}_{n} /\left|z_{n}\right|=1$ if $z_{n}=0$. A fortiori the Myrberg type phenomenon is invalid for $\left(\Delta^{\sim}, \Delta, \pi\right)$ if $(1.3)$ is invalid, completing the proof of Fact 2.

1.4. Throughout this paper we always assume that the determining sequence $\left\{z_{n}\right\}$ of the covering surface $\left(\Delta^{\sim}, \Delta, \pi\right)$ in consideration satisfies (1.3) unless the contrary is explicitly stated. Thus we assume that the Myrberg type phenomenon is valid for $\left(\Delta^{\sim}, \Delta, \pi\right)$. 
A sequence $\left\{\Delta_{n}\right\}$ of closed discs $\Delta_{n}=\bar{\Delta}\left(z_{n}, r_{n}\right)\left(r_{n}>0\right)$ will be referred to as an admissible sequence of discs in $\Delta$ associated with the covering surface $\left(\Delta^{\sim}, \Delta, \pi\right)$, or with its determining sequences $\left\{z_{n}\right\}$, if every $\Delta_{n}$ is contained in $\Delta: \Delta_{n} \subset \Delta(n \geq 1)$, and discs $\Delta_{n}(n \geq 1)$ are disjoint by pairs:

$$
\Delta_{n} \cap \Delta_{m}=\varnothing \quad(n \neq m)
$$

for every positive integers $n$ and $m$. For an admissible sequence $\left\{\Delta_{n}\right\}$ we consider a subregion

$$
D=\Delta \backslash \bigcup_{n \geq 1} \Delta_{n}
$$

of $\Delta$ which gives rise to a smooth covering surface $\left(D^{\sim}, D, \pi\right)$ naturally associated with $\left(\Delta^{\sim}, \Delta, \pi\right)$.

We will study the covering surface $\left(D^{\sim}, D, \pi\right)$ from the view point of the Myrberg type phenomenon.

\section{NONSEPARATING DISCS}

2.1. For an admissible sequence $\left\{\Delta_{n}\right\}$ of discs in $\Delta$ associated with $\left(\Delta^{\sim}, \Delta, \pi\right)$ we consider the subregion $D$ given by (1.4): $D=\Delta \backslash \bigcup_{n \geq 1} \Delta_{n}$. One might feel that the Myrberg type phenomenon does no longer occur for $\left(D^{\sim}, D, \pi\right)$ since all the branch points are removed from $\left(\Delta^{\sim}, \Delta, \pi\right)$ whose presences seemed to be essential for the occurrence of the Myrberg type phenomenon for $\left(\Delta^{\sim}, \Delta, \pi\right)$. On the other hand one might also feel that the Myrberg type phenomenon can occur for $\left(D^{\sim}, D, \pi\right)$ since two sheets of $D^{\sim}$ are equally as tightly joined as $\Delta^{\sim}$ if we take $\left\{\Delta_{n}\right\}$ small enough. It is this latter intuition that is really the case. Namely, we have the following assertion which is the main result of this paper:

Theorem 1. There always exists an admissible sequence $\left\{\Delta_{n}\right\}$ of discs in $\Delta$ associated with any given covering surface $\left(\Delta^{\sim}, \Delta, \pi\right)$ such that the Myrberg type phenomenon occurs for the covering surface $\left(D^{\sim}, D, \pi\right)$.

The proof will be given in 2.2-2.7.

2.2. For any admissible sequence $\left\{\Delta_{k}\right\}$ of discs in $\Delta$ associated with $\left(\Delta^{\sim}, \Delta, \pi\right)$ we denote by

$$
D_{n}=\Delta \backslash \bigcup_{k<n} \Delta_{k} \quad(n=1,2, \ldots),
$$

where we understand that $D_{1}=\Delta$. We denote by $G_{n}(z, a)$ the Green's function on the region $D_{n}(n \geq 1)$ with its pole $a$ in $D_{n}$. In particular $G_{1}(z, a)$ is the Green's function on $D_{1}=\Delta$ so that it can be explicitly given by

$$
G_{1}(z, a)=\log \left|\frac{1-\bar{a} z}{z-a}\right| \text {. }
$$

We first maintain that the following simple inequality holds:

$$
G_{1}(z, a) \geq K(a)(1-|z|),
$$

for every point $z$ in $\Delta$ where $K(a)=(1-|a|)^{2} /(1+|a|)$.

In view of the elementary inequality

$$
\log (1 / \xi) \geq 1-\xi \quad(0 \leq \xi \leq 1),
$$


we proceed as follows:

$$
\begin{aligned}
G_{1}(z, a) & =\log \left|\frac{1-\bar{a} z}{z-a}\right| \geq 1-\left|\frac{z-a}{1-\bar{a} z}\right| \\
& =\left(1-\left|\frac{z-a}{1-\bar{a} z}\right|^{2}\right) /\left(1+\left|\frac{z-a}{1-\bar{a} z}\right|\right) .
\end{aligned}
$$

The numerator of the rightmost side of the above is estimated as

$$
\begin{aligned}
1-\left|\frac{z-a}{1-\bar{a} z}\right|^{2} & =\frac{\left(1-|a|^{2}\right)\left(1-|z|^{2}\right)}{|1-\bar{a} z|^{2}} \\
& \geq \frac{\left(1-|a|^{2}\right)\left(1-|z|^{2}\right)}{(1+|a|)^{2}}=\frac{1-|a|}{1+|a|}\left(1-|z|^{2}\right),
\end{aligned}
$$

and the denominator as

$$
1+\left|\frac{z-a}{1-\bar{a} z}\right| \leq 1+\frac{|z|+|a|}{1-|a|}=\frac{1+|z|}{1-|a|} .
$$

Putting these estimates together we obtain

$$
G_{1}(z, a) \geq \frac{1-|a|}{1+|a|}\left(1-|z|^{2}\right) \cdot \frac{1-|a|}{1+|z|},
$$

which is nothing but (2.2). In our later application of (2.2) the exact shape of $K(a)$ is immaterial except for that it is a finite positive constant dependent only upon $a$.

2.3. We need one more simple inequality. For any positive integer $n \geq 1$ and any point $a$ in $D_{n}$ there exists a finite positive constant $K_{n}(a)$ depending only on $n$ and $a$ such that

$$
G_{n}(z, a) \geq K_{n}(a) G_{1}(z, a),
$$

for every $z=z_{k} \quad(k \geq n)$.

To prove (2.3) we may assume that $n \geq 2$ and we take closed discs $\Delta_{k}^{*}=$ $\bar{\Delta}\left(z_{k}, r_{k}^{*}\right) \quad(1 \leq k \leq n-1)$ such that $r_{k}<r_{k}^{*} \quad(1 \leq k \leq n-1), \Delta_{k}^{*}$ is contained in $\Delta \backslash\left(\left\{z_{j}: j \geq \bar{n}\right\} \cup\{a\}\right) \quad(1 \leq k \leq n-1)$ and $\bar{\Delta}_{k}^{*}(1 \leq k \leq n-1)$ are mutually disjoint. Then we set $D_{n}^{*}=\Delta \backslash \bigcup_{k<n} \Delta_{k}^{*}$. Since $\partial \bar{D}_{n}^{*} \backslash \partial \bar{\Delta}$ is compact in $D_{n}$ and $G_{n}(\cdot, a)>0$ on $D_{n}$, we have

$$
\alpha_{n}(a)=\min \left\{G_{n}(\zeta, a): \zeta \in \partial D_{n}^{*} \backslash \partial \Delta\right\}>0,
$$

and similarly

$$
\beta_{n}(a)=\max \left\{G_{1}(\zeta, a): \zeta \in \partial D_{n}^{*} \backslash \partial \Delta\right\}<\infty .
$$

Then the required $K_{n}(a)$ is given by

$$
K_{n}(a)=\alpha_{n}(a) / \beta_{n}(a) .
$$

In view of $G_{n}(\cdot, a) \leq G_{1}(\cdot, a)$ on $D_{n}$, we have $\alpha_{n}(a) \leq \beta_{n}(a)$ and therefore $0<K_{n}(a) \leq 1$.

The function $u_{n}=G_{n}(\cdot, a)-K_{n}(a) G_{1}(\cdot, a)$ is harmonic on $D_{n}^{*} \backslash\{a\}$ and has a nonnegative singularity at $a$ because of $K_{n}(a) \leq 1$. Therefore $u_{n}$ is a 
superharmonic on $D_{n}^{*}$ and continuous on $\overline{D_{n}^{*}}$ in the extended sense. However $u_{n}(\zeta)=0$ for $\zeta \in \partial \Delta$ and

$$
\begin{aligned}
u_{n}(\zeta) & =G_{n}(\zeta, a)-K_{n}(a) G_{1}(\zeta, a) \\
& =G_{n}(\zeta, a)-\alpha_{n}(a)\left(G_{1}(\zeta, a) / \beta_{n}(a)\right) \\
& \geq G_{n}(\zeta, a)-\alpha_{n}(a) \geq 0,
\end{aligned}
$$

for $\zeta \in \partial D_{n}^{*} \backslash \partial \Delta$. By the maximum principle, $u_{n} \geq 0$ on $D_{n}^{*}$, and in particular $u_{n}\left(z_{k}\right) \geq 0(k \geq n)$, which is nothing but $(2.3)$.

2.4. Let $D_{n}$ be the subregion of $\Delta$ given by (2.1) for each $n \geq 1$ with $\left\{\Delta_{k}\right\}$ an admissible sequence of discs in $\Delta$ associated with $\left(\Delta^{\sim}, \Delta, \pi\right)$.

We claim now that the Myrberg type phenomenon occurs for $\left(D_{n}^{\sim}, D_{n}, \pi\right)$ for every $n \geq 1$ :

$$
H^{\infty}\left(D_{n}^{\sim}\right)=H^{\infty}\left(D_{n}\right) \circ \pi \quad(n=1,2, \ldots) .
$$

Observe that the above assertion for $n=1$ is nothing but the Myrberg type phenomenon for $\left(\Delta^{\sim}, \Delta, \pi\right)$.

Contrary to the assertion assume that there exists a $g$ in $H^{\infty}\left(D_{n}^{\sim}\right) \backslash H^{\infty}\left(D_{n}\right) \circ$ $\pi$. Consider the function $f$ in $H^{\infty}\left(D_{n}\right)$ given by

$$
f(z)=\left(g\left(z^{+}\right)-g\left(z^{-}\right)\right)^{2} \quad\left(z \in D_{n}\right),
$$

where $\pi^{-1}(z)=\left\{z^{+}, z^{-}\right\}$under the convention that $z_{k}^{+}=z_{k}^{-}(k \geq n)$. Since $g$ is not in $H^{\infty}\left(D_{n}\right) \circ \pi$, there exists a point $a$ in $D_{n}$ such that

$$
f(a) \neq 0 \text {. }
$$

On multiplying $g$ by a suitable nonzero constant we can assume that $|f(z)|<1$ $\left(z \in D_{n}\right)$. Using $f$ we define a positive superharmonic function $s$ on $D_{n}$ by

$$
s(z)=-\log |f(z)| \quad\left(z \in D_{n}\right) .
$$

Observe that $s$ has a positive singularity at each $z_{k}$ which is a positive integral multiple of $-\log \left|z-z_{k}\right|$ for each $k \geq n$. Hence

$$
S_{m}(z)=s(z)-\sum_{k=n}^{m} G_{n}\left(z, z_{k}\right)
$$

is superharmonic on $D_{n}$ for any positive integer $m \geq n$. Since $s(z)>0$ on $D_{n}$ and $\sum_{k=n}^{m} G_{n}\left(\zeta, z_{k}\right)=0$ for any $\zeta \in \partial D_{n}$, we have

$$
\liminf _{z \in D_{n}, z \rightarrow \zeta} S_{m}(z) \geq 0
$$

for every $\zeta$ in $\partial D_{n}$ and therefore by the minimum principle $S_{m} \geq 0$ on $D_{n}$ and in particular

$$
s(a) \geq \sum_{k=n}^{m} G_{n}\left(a, z_{k}\right)
$$

for every $m \geq n$. On letting $m \rightarrow \infty$ and then by using (2.3) and (2.2) we obtain

$$
\begin{aligned}
s(a) & \geq \sum_{k \geq n} G_{n}\left(a, z_{k}\right) \geq K_{n}(a) \sum_{k \geq n} G_{1}\left(a, z_{k}\right) \\
& \geq K_{n}(a) K(a) \sum_{k \geq n}\left(1-\left|z_{k}\right|\right) .
\end{aligned}
$$

By (1.3) we must have $s(a)=\infty$ but this is a contradiction since $s(a)=$ $-\log |f(a)|<\infty$ by $(2.5)$. 
2.5. Take an arbitrary admissible sequence $\left\{\Delta_{k}^{*}\right\}$ of discs in $\Delta$ associated with $\left(\Delta^{\sim}, \Delta, \pi\right)$, an arbitrary positive integer $\mu$ and an arbitrary point $a$ in $\Delta \backslash \bigcup_{k \geq 1} \Delta_{k}^{*}$

We set $\pi^{-1}(a)=\left\{a^{+}, a^{-}\right\}$. We claim that there exists an admissible sequence $\left\{\Delta_{k}\right\}$ of discs in $\Delta$ associated with $\left(\Delta^{\sim}, \Delta, \pi\right)$ such that

$$
\begin{cases}\Delta_{k}=\Delta_{k}^{*} & (k<\mu), \\ \Delta_{k} \subset \Delta_{k}^{*} & (k \geq \mu),\end{cases}
$$

where we understand the above as merely $\Delta_{k} \subset \Delta_{k}^{*}(k \geq 1)$ when $\mu=1$, and moreover that $f\left(a^{+}\right)=f\left(a^{-}\right)$, for every $f$ in $H^{\infty}\left(D^{\sim}\right)$ where $D=\Delta \backslash \bigcup_{k \geq 1} \Delta_{k}$ and $\left(D^{\sim}, D, \pi\right)$ is the covering surface of $D$ associated with $\left(\Delta^{\sim}, \Delta, \pi\right)$.

To prove the above assertion we set $\Delta_{k}^{*}=\bar{\Delta}\left(z_{k}, r_{k}^{*}\right)$ for $k \geq \mu$ and consider the disc

$$
\Delta_{k}(m)=\bar{\Delta}\left(z_{k}, r_{k}^{*} / m\right),
$$

for any positive integer $k \geq \mu$ and for any positive integer $m \geq 1$.

Let $\left\{m_{n}\right\} \quad(n \geq \mu)$ be a strictly increasing sequence of positive integers $m_{n}$ which will be specified later. Using this sequence we set $\Delta_{k}=\Delta_{k}^{*} \quad(k<\mu)$ and

$$
\Delta_{k}=\Delta_{k}\left(m_{k}\right)=\bar{\Delta}\left(z_{k}, r_{k}^{*} / m_{k}\right) \quad(k \geq \mu),
$$

and we will show that $\left\{\Delta_{k}\right\}$ is required by choosing $\left\{m_{k}\right\} \quad(k \geq \mu)$ suitably. Still keeping $\left\{m_{k}\right\} \quad(k \geq \mu)$ unfixed we set

$$
D_{n}=\Delta \backslash \bigcup_{k<n} \Delta_{k} \quad(n \geq \mu),
$$

where we understand the above as $D_{1}=\Delta$ when $\mu=1$ and $n=1$, and also we set

$$
D_{n}(m)=\Delta \backslash\left(\left(\bigcup_{k<n} \Delta_{k}\right) \cup\left(\bigcup_{k \geq n} \Delta_{k}(m)\right)\right) \quad(n \geq \mu)
$$

for every positive integer $m$.

We denote by $A_{n}(m) \quad(n \geq \mu)$ the family of functions $f$ in $H^{\infty}\left(\pi^{-1}\left(D_{n}(m)\right)\right)$ such that $f\left(a^{-}\right)=0$ and

$$
\|f\|=\|f\|_{\pi^{-1}\left(D_{n}(m)\right)} \leq 1,
$$

and we consider the extremum problem:

$$
M_{n}(m)=\sup \left\{\left|f\left(a^{+}\right)\right|: f \in A_{n}(m)\right\}<\infty .
$$

We then maintain the following:

$$
M_{n}(m) \downarrow 0 \quad(m \uparrow \infty) .
$$

In fact, $A_{n}(m) \supset A_{n}(m+1)$ and a fortiori $M_{n}(m) \geq M_{n}(m+1) \quad(m \geq 1)$. Contrary to the assertion assume that

$$
\inf _{m \geq 1} M_{n}(m)=\varepsilon>0 .
$$

For any positive integer $\nu$ we can find a $g_{\nu}$ in $A_{n}(m)$ such that $M_{n}(m) \geq$ $\left|g_{\nu}\left(a^{+}\right)\right| \geq M_{n}(m)-1 / \nu$.

Since $\left\{g_{\nu}\right\}$ forms a normal family on $\pi^{-1}\left(D_{n}(m)\right)$, there exists a subsequence $\left\{g_{\nu^{\prime}}\right\}$ of $\left\{g_{\nu}\right\}$ such that $\left\{g_{\nu^{\prime}}\right\}$ converges to an $f_{m}$ uniformly on each 
compact subset of $\pi^{-1}\left(D_{n}(m)\right)$ so that $f_{m} \in A_{n}(m)$ and $\left|f_{m}\left(a^{+}\right)\right|=M_{n}(m)$. Again $\left\{f_{m}\right\}$ forms a normal family on the subdomain

$$
X=D_{n}^{\sim} \backslash \pi^{-1}\left(\left\{z_{k}: k \geq n\right\}\right)
$$

of $D_{n}^{\sim}$ and therefore there exists a subsequence $\left\{f_{m^{\prime}}\right\}$ of $\left\{f_{m}\right\}$ such that $\left\{f_{m^{\prime}}\right\}$ converges to an $f$ uniformly on each compact subset of $X$ so that $f \in H^{\infty}(X)$ and

$$
\left|f\left(a^{+}\right)\right|=\lim _{m^{\prime} \rightarrow \infty}\left|f_{m^{\prime}}\left(a^{+}\right)\right|=\lim _{m^{\prime} \rightarrow \infty} M_{n}\left(m^{\prime}\right)=\varepsilon
$$

and similarly

$$
f\left(a^{-}\right)=\lim _{m^{\prime} \rightarrow \infty} f_{m^{\prime}}\left(a^{-}\right)=0 .
$$

Since $\pi^{-1}\left(\left\{z_{k}: k \geq n\right\}\right)$ is discrete in $D_{n}^{\sim}$ and $f$ is bounded on $X$, the Riemann removability theorem assures that $f$ can be continued to $D_{n}^{\sim}$ so that $f \in H^{\infty}\left(D_{n}^{\sim}\right)$. As we saw above, $f\left(a^{+}\right) \neq 0$ and $f\left(a^{-}\right)=0$ and therefore $f$ does not belong to $H^{\infty}\left(D_{n}\right) \circ \pi$. This contradicts (2.4) and the proof for (2.6) is herewith complete.

We are now in a position to determine $\left\{m_{n}\right\} \quad(n \geq \mu)$ inductively. First, by (2.6), we can choose a positive integer $m_{\mu}$ such that $M_{\mu}\left(m_{\mu}\right)<1 / \mu$.

Suppose we have chosen positive integers $m_{j}(\mu \leq j \leq n)$ such that $m_{j}<$ $m_{k} \quad(j<k)$ and $M_{j}\left(m_{j}\right)<1 / j \quad(\mu \leq j \leq n)$.

Then, again by (2.6), we can find a positive integer $m_{n+1}>m_{n}$ such that

$$
M_{n+1}\left(m_{n+1}\right)<1 /(n+1) .
$$

Finally we show that $f\left(a^{+}\right)=f\left(a^{-}\right)$for any $f$ in $H^{\infty}\left(D^{\sim}\right)$. Observe that

$$
D=\Delta \backslash \bigcup_{k \geq 1} \Delta_{k} \supset D_{n}\left(m_{n}\right) \quad(n \geq \mu)
$$

and therefore

$$
D^{\sim} \supset \pi^{-1}\left(D_{n}\left(m_{n}\right)\right) \quad(n \geq \mu) .
$$

Hence if we set $g=c\left(f-f\left(a^{-}\right)\right)$with a positive constant $c$, then $g$ is bounded and holomorphic on $D^{\sim}$ along with $f$ and a fortiori

$$
g \in H^{\infty}\left(\pi^{-1}\left(D_{n}\left(m_{n}\right)\right)\right) \quad(n \geq \mu) .
$$

We may assume that $\|g\|_{D^{\sim}}<1$ by taking the positive constant $c$ small enough. This with $g\left(a^{-}\right)=0$ assures that $g \in A_{n}\left(m_{n}\right) \quad(n \geq \mu)$.

Therefore we have to have

$$
\left|g\left(a^{+}\right)\right| \leq M_{n}\left(m_{n}\right)<1 / n \quad(n \geq \mu),
$$

which yields that $g\left(a^{+}\right)=0$ or $f\left(a^{+}\right)=f\left(a^{-}\right)$.

2.6. By Fact 1 we can now conclude that the Myrberg type phenomenon occurs for $\left(D^{\sim}, D, \pi\right)$ and thus the proof of Theorem 1 is over at this stage.

Nevertheless we will prove in the next section the relation $H^{\infty}\left(D^{\sim}\right)=H^{\infty}(D)$ $\circ \pi$ based upon the result in 2.5 without appealing with Fact 1 . The reason why we do this is to suggest a further generalization of our result to the case where we replace $D$ by a general Riemann surface for which any counterpart of Fact 1 cannot be expected. 
2.7. We now prove $H^{\infty}\left(D^{\sim}\right)=H^{\infty}(D) \circ \pi$ based upon the result obtained in 2.5 without using Fact 1.

First we fix an auxiliary admissible sequence $\left\{\Delta_{0 k}\right\}$ of discs in $\Delta$ associated with $\left(\Delta^{\sim}, \Delta, \pi\right)$. Fix an arbitrary sequence $\left\{a_{\mu}\right\}(\mu=1,2, \ldots)$ of distinct points in $X_{0}=\Delta \backslash \bigcup_{k>1} \Delta_{0 k}$ convergent to a point $a_{\infty}$ in $X_{0}$.

By using the result in 2.5 we construct an admissible sequence $\left\{\Delta_{\mu k}\right\}$ in $\Delta$ for each positive integer $\mu \geq 1$ such that

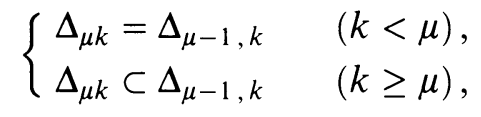

and $f\left(a_{\mu}^{+}\right)=f\left(a_{\mu}^{-}\right)$for any $f$ in $H^{\infty}\left(D_{\mu}^{\sim}\right)$ where $D_{\mu}=\Delta \backslash \bigcup_{k>1} \Delta_{\mu k}$, and $\left(D_{\mu}^{\sim}, D_{\mu}, \pi\right)$ is a covering surface associated with $\left(\Delta^{\sim}, \Delta, \pi\right)$. The relations among $\Delta_{\mu k}$ are as indicated in the following diagram:

$$
\begin{aligned}
& \Delta_{01} \supset \Delta_{11}=\Delta_{21}=\Delta_{31}=\Delta_{41}=\Delta_{51} \cdots \\
& \Delta_{02} \supset \Delta_{12} \supset \Delta_{22}=\Delta_{32}=\Delta_{42}=\Delta_{52} \cdots \\
& \Delta_{03} \supset \Delta_{13} \supset \Delta_{23} \supset \Delta_{33}=\Delta_{43}=\Delta_{53} \cdots \\
& \Delta_{04} \supset \Delta_{14} \supset \Delta_{24} \supset \Delta_{34} \supset \Delta_{44}=\Delta_{54} \cdots \\
& \Delta_{05} \supset \Delta_{15} \supset \Delta_{25} \supset \Delta_{35} \supset \Delta_{45} \supset \Delta_{55} \cdots
\end{aligned}
$$

We now define the required admissible sequence $\left\{\Delta_{n}\right\}$ of discs in $\Delta$ associated with $\left(\Delta^{\sim}, \Delta, \pi\right)$ as follows:

$$
\Delta_{n}=\Delta_{n n} \quad(n \geq 1) .
$$

By our construction we see that $\Delta_{n} \subset \Delta_{\mu n}(n \geq 1)$ for every positive integer $\mu \geq 1$. Therefore

$$
D_{\mu}=\Delta \backslash \bigcup_{k \geq 1} \Delta_{\mu k} \subset D=\Delta \backslash \bigcup_{k \geq 1} \Delta_{k} \quad(\mu \geq 1),
$$

and hence $D_{\mu}^{\sim} \subset D^{\sim}(\mu \geq 1)$. Take any $f$ in $H^{\infty}\left(D^{\sim}\right)$. Since $H^{\infty}\left(D^{\sim}\right) \subset$ $H^{\infty}\left(D_{\mu}^{\sim}\right)$ for every $\mu \geq 1$, we see that

$$
f\left(a_{\mu}^{+}\right)=f\left(a_{\mu}^{-}\right) \quad(\mu \geq 1) .
$$

For any $f$ in $H^{\infty}\left(D^{\sim}\right)$ we set

$$
g(z)=\left(f\left(z^{+}\right)-f\left(z^{-}\right)\right)^{2} \quad(z \in D),
$$

where $\pi^{-1}(z)=\left\{z^{+}, z^{-}\right\}$. In view of (2.7) we see that $g\left(a_{\mu}\right)=0(\mu \geq 1)$. Since $\left\{a_{\mu}\right\}$ is a sequence in $D$ convergent to a point $a_{\infty}$ in $D$, the unicity theorem assures that $g \equiv 0$ on $D$ and therefore $f \in H^{\infty}(D) \circ \pi$, i.e., the Myrberg type phenomenon occurs for $\left(D^{\sim}, D, \pi\right)$.

The proof of Theorem 1 started from $\S 2.2$ is herewith complete.

2.8. An admissible sequence $\left\{\Delta_{k}\right\}$ of discs in $\Delta$ associated with $\left(\Delta^{\sim}, \Delta, \pi\right)$ will be said to be nonseparating (separating, resp.) for $\left(\Delta^{\sim}, \Delta, \pi\right)$ if the Myrberg type phenomenon occurs (does not occur, resp.) for $\left(D^{\sim}, D, \pi\right)$ where $D=\Delta \backslash \bigcup_{k \geq 1} \Delta_{k}$. The main result (Theorem 1) of this section may be then 
restated as follows: There always exists a nonseparating sequence of discs in $\Delta$ for any $\left(\Delta^{\sim}, \Delta, \pi\right)$.

Take two admissible sequences $\left\{\Delta_{k}\right\}$ and $\left\{\Delta_{k}^{\prime}\right\}$ of discs in $\Delta$ associated with $\left(\Delta^{\sim}, \Delta, \pi\right)$. We may say that $\left\{\Delta_{k}\right\}$ is smaller (larger, resp.) than $\left\{\Delta_{k}^{\prime}\right\}$ if $\Delta_{k} \subset \Delta_{k}^{\prime}\left(\Delta_{k} \supset \Delta_{k}^{\prime}\right.$, resp.) for every $k \geq 1$. We clearly have the following: If $\left\{\Delta_{k}\right\}$ is a nonseparating sequence of discs for $\left(\Delta^{\sim}, \Delta, \pi\right)$ and $\left\{\Delta_{k}^{\prime}\right\}$ is smaller than $\left\{\Delta_{k}\right\}$, then $\left\{\Delta_{k}^{\prime}\right\}$ is also a nonseparating sequence of discs for $\left(\Delta^{\sim}, \Delta, \pi\right)$.

\section{RIGID COVERING SURFACES}

3.1. In $\S 2$ we have seen that the Myrberg type phenomenon occurs for the covering surface $\left(D^{\sim}, D, \pi\right)$ of $D=\Delta \backslash \bigcup_{k \geq 1} \Delta_{k}$ associated with any covering surface $\left(\Delta^{\sim}, \Delta, \pi\right)$ for which the Myrberg type phenomenon occurs if $\left\{\Delta_{k}\right\}$ is taken small enough. In other words, $\left\{\Delta_{k}\right\}$ is nonseparating if $\left\{\Delta_{k}\right\}$ is taken small enough. One might expect, conversely, that we can have the situation that the Myrberg type phenomenon does not occur for $\left(D^{\sim}, D, \pi\right)$ by taking $\left\{\Delta_{k}\right\}$ large enough. In other words, we expect that $\left\{\Delta_{k}\right\}$ can always be made separating by taking $\left\{\Delta_{k}\right\}$ suitably. Rather unexpectedly at the first sight we have the following result.

Theorem 2. There exists a covering surface $\left(\Delta^{\sim}, \Delta, \pi\right)$ such that any admissible sequence $\left\{\Delta_{k}\right\}$ of discs in $\Delta$ associated with $\left(\Delta^{\sim}, \Delta, \pi\right)$ is always nonseparating.

It would be impressive to call a covering surface $\left(\Delta^{\sim}, \Delta, \pi\right)$ rigid if it has the property stated in the above theorem. If $\left(\Delta^{\sim}, \Delta, \pi\right)$ is not rigid, then it will then be called nonrigid.

The proof of Theorem 2 is, however, very simple and will be given in 3.2-3.3. Actually we will assert a bit more than stated in the above theorem.

3.2. Choose an arbitrary two sheeted covering surface $\left(\Delta^{* \sim}, \Delta, \pi^{*}\right)$ of the open unit disc $\Delta$ for which the Myrberg type phenomenon occurs. We will show that it can be made rigid by further adding branch points. The addition is done in such a fashion that $t w o$ new branch points are provided to each original branch point placed very close to it. Here the number $t w o$ of added new branch points is inessential. We can add as many new branch points as we wish and moreover the number may vary from original branch point to point as far as the number is kept even. Only for the sake of simplicity we restrict the number to be two.

To perform the procedure mentioned above explicitly we denote by $\left\{z_{3 k-1}\right\}$ $(k=1,2, \ldots)$ the determining sequence of $\left(\Delta^{* \sim}, \Delta, \pi^{*}\right)$, i.e., $\left\{\pi^{*-1}\left(z_{3 k-1}\right)\right.$ : $k=1,2, \ldots\}$ is the totality of branch points of $\left(\Delta^{* \sim}, \Delta, \pi^{*}\right)$ so that

$$
\sum_{k \geq 1}\left(1-\left|z_{3 k-1}\right|\right)=\infty
$$

By Theorem 1 we can find a nonseparating sequence $\left\{\Delta_{k}^{*}\right\}$ of discs in $\Delta$ associated with $\left(\Delta^{* \sim}, \Delta, \pi^{*}\right)$. Let $\Delta_{k}^{*}=\bar{\Delta}\left(z_{3 k-1}, r_{k}^{*}\right) \quad(k=1,2, \ldots)$, and

$$
D^{*}=\Delta \backslash \bigcup_{k \geq 1} \Delta_{k}^{*}
$$


Recall that $\left\{\Delta_{k}^{*}\right\}$ is nonseparating means that

$$
H^{\infty}\left(\pi^{*-1}\left(D^{*}\right)\right)=H^{\infty}\left(D^{*}\right) \circ \pi^{*} .
$$

Choose two arbitrary distinct points $z_{3 k-2}$ and $z_{3 k}$ in the disc $\Delta\left(z_{3 k-1}, r_{k}^{*} / 2\right)$ different from $z_{3 k-1}$ :

$$
\left\{z_{3 k-2}, z_{3 k}\right\} \subset \Delta\left(z_{3 k-1}, r_{k}^{*} / 2\right) \backslash\left\{z_{3 k-1}\right\} .
$$

We then consider the covering surface $\left(\Delta^{\sim}, \Delta, \pi\right)$ whose determining sequence is

$$
\left\{z_{n}: n=1,2, \ldots\right\}=\left\{z_{3 k-2}, z_{3 k-1}, z_{3 k}: k=1,2, \ldots\right\} .
$$

For simplicity we will, and actually we may, make the following identification:

$$
\pi^{-1}\left(D^{*}\right)=\pi^{*-1}\left(D^{*}\right)
$$

The relation (3.2) implies, by (3.4) above, that

$$
H^{\infty}\left(\pi^{-1}\left(D^{*}\right)\right)=H^{\infty}\left(D^{*}\right) \circ \pi .
$$

3.3. We now show that the covering surface $\left(\Delta^{\sim}, \Delta, \pi\right)$ constructed in 3.2 is rigid. For the purpose take an arbitrary admissible sequence $\left\{\Delta_{n}\right\} \quad(n=$ $1,2, \ldots)$ of discs in $\Delta$ associated with $\left(\Delta^{\sim}, \Delta, \pi\right)$. Because of $(3.3)$ we have

$$
\Delta_{3 k-2} \cup \Delta_{3 k-1} \cup \Delta_{3 k} \subset \Delta_{k}^{*} \quad(k=1,2, \ldots),
$$

no matter how we choose $\left\{\Delta_{k}\right\}$. This extremely simple fact, however, turns out to be the key point that makes $\left(\Delta^{\sim}, \Delta, \pi\right)$ rigid. Let $D=\Delta \backslash \bigcup_{n \geq 1} \Delta_{n}$. We need to show that the Myrberg type phenomenon occurs for $\left(D^{\sim}, D, \pi\right)$ associated with $\left(\Delta^{\sim}, \Delta, \pi\right)$.

By (3.6) we have to have $D \supset D^{*}$ and hence

$$
H^{\infty}\left(D^{\sim}\right)=H^{\infty}\left(\pi^{-1}(D)\right) \subset H^{\infty}\left(\pi^{-1}\left(D^{*}\right)\right) .
$$

Take an arbitrary $f$ in $H^{\infty}\left(D^{\sim}\right)$ and set

$$
g(z)=\left(f\left(z^{+}\right)-f\left(z^{-}\right)\right)^{2} \quad(z \in D),
$$

where $\pi^{-1}(z)=\left\{z^{+}, z^{-}\right\}$. Since $f \in H^{\infty}\left(\pi^{-1}\left(D^{*}\right)\right)$ by (3.7), we have, by (3.5), $g \equiv 0$ on $D^{*}(\subset D)$ and a fortiori on $D$ which means that $f \in H^{\infty}(D)$ 。 $\pi$. We have shown $H^{\infty}\left(D^{\sim}\right)=H^{\infty}(D) \circ \pi$ without using Fact 1, i.e., the Myrberg type phenomenon occurs for $\left(D^{\sim}, D, \pi\right)$.

The proof of Theorem 2 is herewith complete.

\section{NONRIGID COVERING SURFACES}

4.1. In spite of the fact that we have seen previously in $\S 3$ the existence of rigid covering surfaces, we still tend to feel it ordinary that almost all covering surfaces are nonrigid. However to exhibit some nonrigid covering surface seems to be nontrivial and hence we state the following:

Theorem 3. There exists a covering surface $\left(\Delta^{\sim}, \Delta, \pi\right)$ such that there exists an admissible sequence $\left\{\Delta_{k}\right\}$ of discs in $\Delta$ associated with $\left(\Delta^{\sim}, \Delta, \pi\right)$ which is separating.

Recall that we called a covering surface $\left(\Delta^{\sim}, \Delta, \pi\right)$ with the property stated in the above theorem to be nonrigid and an admissible sequence $\left\{\Delta_{k}\right\}$ to be 
separating if $H^{\infty}\left(D^{\sim}\right)$ separates the points in the fiber $\pi^{-1}(z)$ for at least one point $z$ in $D$ where $D=\Delta \backslash \bigcup_{k>1} \Delta_{k}$ and $\left(D^{\sim}, D, \pi\right)$ is the covering surface associated with $\left(\Delta^{\sim}, \Delta, \pi\right)$. If we use Fact 1 stated in $\S 1.1$, then we can replace "for at least one" in the above statement by "for all." In our proof of Theorem 3 that will be given in 4.2-4.4 we will directly show that $H^{\infty}\left(D^{\sim}\right)$ separates the points in the fibers $\pi^{-1}(z)$ for all points $z$ in $D$ without appealing to Fact 1 .

Given an arbitrary two sheeted covering surface $\left(\Delta^{* \sim}, \Delta, \pi^{*}\right)$ for which the Myrberg type phenomenon occurs. We could reform it in 3.2 to be rigid by adding two new branch points arbitrarily placed close enough to each original branch point. We will also reform $\left(\Delta^{* \sim}, \Delta, \pi^{*}\right)$ to be nonrigid by adding one new branch point arbitrarily placed close enough to each original branch point.

The numbers two and one in the above additions are quite suggestive. The effects of the additions of two or one point may be explained as follows.

Consider a two sheeted covering surface $\Delta(a, r)^{\sim}$ of a disc $\Delta(a, r)$ with a single branch point over $a$ and also consider the circle $\gamma=\partial \Delta(a, r / 2)$. The counter image $\gamma^{\sim}$ of $\gamma$ in $\Delta(a, r)^{\sim}$ is connected. If we add two branch points to $\Delta(a, r)^{\sim}$ over arbitrary distinct two points in $\Delta(a, r / 2) \backslash\{a\}$, then the counter image $\gamma^{\sim}$ of $\gamma$ in the new $\Delta(a, r)^{\sim}$ with three branch points is still connected. However, if we add one branch point to the original $\Delta(a, r)^{\sim}$ over an arbitrary point in $\Delta(a, r / 2) \backslash\{a\}$, then the counter image $\gamma^{\sim}$ of $\gamma$ in the reformed $\Delta(a, r)^{\sim}$ with two branch points is not connected.

The above observation suggests the following. The first procedure of adding two points each to each original branch point gives the reformed covering surface a tendency to become to be nonseparated in a certain sense. While by the second procedure of adding one point the reformed covering surface is made to tend to be separated in a suitable sense.

4.2. To prove Theorem 3 we choose an arbitrary two sheeted covering surface $\left(\Delta^{*} \sim, \Delta, \pi^{*}\right)$ whose determining sequence in $\Delta$ is $\left\{z_{2 n}: n=1,2, \ldots\right\}$ with

$$
\sum_{n \geq 1}\left(1-\left|z_{2 n}\right|\right)=\infty
$$

We will give a new sequence $\left\{z_{2 n-1}\right\}$ in $\Delta$ such that $z_{2 n-1}$ is arbitrarily placed to very close to $z_{2 n}$ for each $n$. We then reform $\left(\Delta^{* \sim}, \Delta, \pi^{*}\right)$ to the required $\left(\Delta^{\sim}, \Delta, \pi\right)$ by adding one new branch point each over $z_{2 n-1}$ for each $n \geq 1$.

To accomplish the goal mentioned above we first choose a sequence $\left\{\alpha_{n}\right\}$ of real numbers $\alpha_{n}$ such that

$$
0<\alpha_{n}<\min \left(\inf _{k \neq n}\left|z_{2 k}-z_{2 n}\right|, 1-\left|z_{2 n}\right|\right) / 2 \quad(n=1,2, \ldots),
$$

and

$$
\sum_{n \geq 1} \alpha_{n}<\infty
$$

Since $0<\alpha_{n}<1$, we have $\alpha_{n}^{2}<\alpha_{n}$ and therefore $\bar{\Delta}\left(z_{2 n}, \alpha_{n}^{2}\right) \quad(n \geq 1)$ are mutually disjoint and contained in $\Delta$. that

We choose arbitrarily and then fix a sequence $\left\{z_{2 n-1}: n \geq 1\right\}$ in $\Delta$ such

$$
z_{2 n-1} \in \Delta\left(z_{2 n}, \alpha_{n}^{2}\right) \backslash\left\{z_{2 n}\right\} \quad(n \geq 1)
$$


We can now consider a new covering surface $\left(\Delta^{\sim}, \Delta, \pi\right)$ whose determining sequence is $\left\{z_{n}: n \geq 1\right\}$ which is the union of the original sequence $\left\{z_{2 n}\right.$ : $n \geq 1\}$ and the new sequence $\left\{z_{2 n-1}: n \geq 1\right\}$. Because of (4.1) we clearly have $\sum_{n \geq 1}\left(1-\left|z_{n}\right|\right)=\infty$ so that the Myrberg type phenomenon occurs for $\left(\Delta^{\sim}, \Delta, \pi\right)$.

To see that $\left(\Delta^{\sim}, \Delta, \pi\right)$ is required we need to find an admissible sequence $\left\{\Delta_{k}\right\}$ of discs in $\Delta$ associated with $\left(\Delta^{\sim}, \Delta, \pi\right)$ which is separating. For the purpose set

$$
\beta_{n}=\left|z_{2 n}-z_{2 n-1}\right| / 4 \quad(n \geq 1),
$$

so that, by (4.4), $4 \beta_{n}<\alpha_{n}^{2}<\alpha_{n}$. Using the sequence $\left\{\beta_{n}: n \geq 1\right\}$ we set

$$
\left\{\begin{array}{l}
\Delta_{2 n}=\bar{\Delta}\left(z_{2 n}, 2 \beta_{n}\right), \\
\Delta_{2 n-1}=\bar{\Delta}\left(z_{2 n-1}, \beta_{n}^{\prime}\right),
\end{array}\right.
$$

for $n \geq 1$, where $\beta_{n}^{\prime}$ may be anything as long as it satisfies $0<\beta_{n}^{\prime} \leq \beta_{n}$ and we will set $\beta_{n}^{\prime}=\beta_{n}$, for example, just for definiteness. Then we put $D=\Delta \backslash \bigcup_{k \geq 1} \Delta_{k}$, and consider the covering surface $\left(D^{\sim}, D, \pi\right)$ associated with $\left(\Delta^{\sim}, \Delta, \pi\right)$.

Recall that $\left\{\Delta_{k}\right\}$ is separating if $H^{\infty}\left(D^{\sim}\right)$ separates the points in $\pi^{-1}(z)$ for at least one $z$ in $D$. We will actually construct in $\S 4.3$ an $f$ in $H^{\infty}\left(D^{\sim}\right)$ such that $f$ separates the points in $\pi^{-1}(z)$ for every $z$ in $D$.

4.3. Consider the infinite product

$$
p(z)=\prod_{n \geq 1}\left(1-q_{n}(z)\right),
$$

where

$$
q_{n}(z)=\frac{z_{2 n-1}-z_{2 n}}{z-z_{2 n}}
$$

so that

$$
p(z)=\prod_{n \geq 1} \frac{z-z_{2 n-1}}{z-z_{2 n}} .
$$

To see the convergence of $(4.5)$ in $\Delta$ observe that

$$
\left|q_{n}(z)\right| \leq 4 \beta_{n} / d_{m}(z) \leq \alpha_{n} / d_{m}(z) \quad(n \geq m),
$$

where $d_{m}(z)$ is the distance between $z$ and $\left\{z_{2 k}: k \geq m\right\} \quad(m \geq 1)$. Since $d_{m}(z)$ is continuous on $\Delta$ and strictly positive on $\Delta \backslash\left\{z_{2 k}: k \geq m\right\}$ we see that $\sum_{n \geq m} q_{n}(z)$ converges absolutely and uniformly on each compact subset of $\Delta \backslash\left\{z_{2 k}: k \geq m\right\}$. Therefore $p(z)$ defines a meromorphic function on $\Delta$ and, by (4.7), $\left\{z_{2 n}: n \geq 1\right\}$ is the set of poles of $p(z)$ in $\Delta$ and $\left\{z_{2 n-1}\right.$ : $n \geq 1\}$ the set of zeros of $p(z)$ on $\Delta$. Thus the square root $\sqrt{p(z)}$ defines a single-valued meromorphic function $\sqrt{p}$ on $\Delta^{\sim}$ whose pole set is $\left\{\pi^{-1}\left(z_{2 n}\right)\right.$ : $n \geq 1\}$ and zero set $\left\{\pi^{-1}\left(z_{2 n-1}\right): n \geq 1\right\}$ so that the restriction $f$ of $\sqrt{p}$ on $D^{\sim}$ is a holomorphic function on $D^{\sim}$ without zeros. Since

$$
f\left(z^{+}\right)=\sqrt{p}\left(z^{+}\right)=-\sqrt{p}\left(z^{-}\right)=-f\left(z^{-}\right) \neq 0
$$

for every $z$ in $D$ where $\pi^{-1}(z)=\left\{z^{+}, z^{-}\right\}$, we see that $f$ separates the points in the fiber $\pi^{-1}(z)$ for every $z$ in $D$. 
4.4. The proof will be over if we show that $f$ belongs to $H^{\infty}\left(D^{\sim}\right)$ or what amounts to the same $\sqrt{p}$ is bounded on $D^{\sim}$ or equivalently $p$ is bounded on $D$. For the purpose we only have to show that $p$ is bounded on $X=$ $\Delta \backslash \bigcup_{n \geq 1} \Delta_{2 n}$, since $X \supset D$. Fix an arbitrary $a \in X$ and we will show that

$$
|p(a)| \leq A
$$

where $A=3 \prod_{n \geq 1}\left(1+\alpha_{n}\right)<\infty$ by (4.3), which will complete the proof.

Fix an arbitrary positive integer $m \geq 1$ and set

$$
Y_{m}=\mathbf{C}^{\wedge} \backslash \bigcup_{1 \leq n \leq m} \Delta_{2 n}
$$

where $\mathbf{C}^{\wedge}$ is the extended complex plane. We also set

$$
p_{m}(z)=\prod_{1 \leq n \leq m}\left(1-q_{n}(z)\right),
$$

which is holomorphic on the closure $\bar{Y}_{m}$ of $Y_{m}$ in the extended plane.

Take an arbitrary $\zeta$ in $\partial \Delta_{2 k} \quad(1 \leq k \leq m)$. If $n=k$, then by the choice of $\beta_{k}$ and $\Delta_{2 k}$,

$$
\left|q_{n}(\zeta)\right|=\left|q_{k}(\zeta)\right|=4 \beta_{k} / 2 \beta_{k}=2,
$$

and therefore

$$
\left|1-q_{k}(\zeta)\right| \leq 3 \leq 3\left(1+\alpha_{k}\right) \quad\left(\zeta \in \partial \Delta_{2 k}\right) .
$$

If $n \neq k \quad(1 \leq n \leq m)$, then by (4.2) with others

$$
\left|q_{n}(\zeta)\right| \leq 4 \beta_{n} / \alpha_{n} \leq \alpha_{n}^{2} / \alpha_{n}=\alpha_{n},
$$

and a fortiori

$$
\left|1-q_{n}(\zeta)\right| \leq 1+\alpha_{n} \quad\left(\zeta \in \partial \Delta_{2 k}, 1 \leq n \leq m, n \neq k\right) .
$$

This with (4.9) now implies that

$$
\begin{aligned}
\left|p_{m}(\zeta)\right| & =\prod_{1 \leq n \leq m}\left|1-q_{n}(\zeta)\right| \\
& \leq\left|1-q_{k}(\zeta)\right| \cdot\left(\prod_{1 \leq n \leq m, n \neq k}\left|1-q_{n}(\zeta)\right|\right) \\
& \leq 3\left(1+\alpha_{k}\right) \cdot\left(\prod_{1 \leq n \leq m, n \neq k}\left(1+\alpha_{n}\right)\right) \\
& =3 \prod_{1 \leq n \leq m}\left(1+\alpha_{n}\right) \leq A
\end{aligned}
$$

for every $\zeta$ in $\partial \Delta_{2 k} \quad(1 \leq k \leq m)$. Since $\partial Y_{m}=\bigcup_{1 \leq k \leq m} \partial \Delta_{2 k}$, we have

$$
\max _{\zeta \in \partial Y_{m}}\left|p_{m}(\zeta)\right| \leq A
$$

By the maximum modulus principle, $\left|p_{m}(z)\right| \leq A$ for all $z$ in $Y_{m}$ and in particular $\left|p_{m}(a)\right| \leq A$. In view of $p_{m}(a) \rightarrow p(a) \quad(m \rightarrow \infty)$, we can conclude (4.8).

The proof of Theorem 3, started in $\S 4.2$, is herewith complete. 


\section{SeParating Ellipses}

5.1. Both the existence of rigid covering surfaces and the nontriviality of the existence of nonrigid covering surfaces come from the particular geometric shape of removing figures as being discs. If we allow ellipses instead of discs as removing figures, then the situation turns out to be drastically simple as is seen in the sequel.

By a closed ellipse (or simply an ellipse) $\delta$ about a point $a$ we mean a closed region bounded by an ellipse $\partial \delta$ (as a curve) one of whose foci is $a$. A sequence $\left\{\delta_{k}\right\}$ of closed ellipses $\delta_{k}$ about $z_{k}$ will be referred to as an admissible sequence of ellipses in $\Delta$ associated with a covering surface $\left(\Delta^{\sim}, \Delta, \pi\right)$ whose determining sequence is $\left\{z_{k}\right\}$ if every ellipse $\delta_{k}$ is contained in $\Delta: \delta_{k} \subset \Delta$ $(k=1,2, \ldots)$, ellipses $\delta_{k}(k \geq 1)$ are mutually disjoint:

$$
\delta_{n} \cap \delta_{m}=\varnothing \quad(n \neq m),
$$

for all positive integers $n$ and $m$, and diameters of $\delta_{k}$ which are nothing but the lengths of major axes of $\delta_{k}$, denoted by $\operatorname{diam} \delta_{k}$, converge to zero:

$$
\lim _{k \rightarrow \infty} \operatorname{diam} \delta_{k}=0 \text {. }
$$

Despite the existence of rigid covering surfaces of the open unit disc $\Delta$ (Theorem 2) we have the following:

Theorem 4. For any two sheeted covering surface $\left(\Delta^{\sim}, \Delta, \pi\right)$, there always exists an admissible sequence $\left\{\delta_{k}\right\}$ of ellipses in $\Delta$ associated with $\left(\Delta^{\sim}, \Delta, \pi\right)$ such that $H^{\infty}\left(d^{\sim}\right)$ separates the points in the covering surface $d^{\sim}$ of

$$
d=\Delta \backslash \bigcup_{k \geq 1} \delta_{k}
$$

associated with $\left(\Delta^{\sim}, \Delta, \pi\right)$ so that the Myrberg type phenomenon does not occur for $\left(d^{\sim}, d, \pi\right)$.

In other words, every covering surface $\left(\Delta^{\sim}, \Delta, \pi\right)$ is always nonrigid in the weak sense.

The proof will be given in 5.2-5.5.

5.2. For any two points $\alpha$ and $\beta$ in $\mathbf{C}$ we denote by $[\alpha, \beta]$ the line segment whose end points are $\alpha$ and $\beta:[\alpha, \beta]=\{(1-t) \alpha+t \beta: 0 \leq t \leq 1\}$. We first show the following:

With any discrete sequence $\left\{z_{n}\right\}$ in $\Delta$ we can associate a discrete sequence $\left\{w_{n}\right\}$ in $\Delta \backslash\{0\}$ such that

$$
\begin{gathered}
\sum_{n \geq 1}\left(1-\left|w_{n}\right|\right)<\infty, \\
{\left[z_{n}, w_{n}\right] \cap\left[z_{m}, w_{m}\right]=\varnothing \quad(n \neq m)}
\end{gathered}
$$

for all positive integers $n$ and $m$ and

$$
\lim _{n \rightarrow \infty}\left|z_{n}-w_{n}\right|=0 \text {. }
$$

In application of this assertion in our proof of Theorem $4,\left\{z_{n}\right\}$ will be the determining sequence of the given covering surface $\left(\Delta^{\sim}, \Delta, \pi\right)$ and the sequence $\left\{w_{n}\right\}$ obtained determines a new two sheeted covering surface of $\Delta$ 
of the exceptional character, i.e., the contrary to (5.1) has been postulated for our covering surfaces in considerations thus far. The procedure in the above assertion may be termed as the sweeping out of branch points of $\left(\Delta^{\sim}, \Delta, \pi\right)$.

By renumbering the sequence $\left\{z_{n}\right\}$, if necessary, we can assume that $\left\{\left|z_{n}\right|\right\}$ is nondecreasing: $\left|z_{1}\right| \leq\left|z_{2}\right| \leq \cdots \leq\left|z_{n}\right| \leq\left|z_{n+1}\right| \leq \cdots$. We denote by $P$ the sequence $\left\{z_{n}\right\}$ as a set. To construct $\left\{w_{n}\right\}$ we first fix a strictly increasing sequence $\left\{t_{n}\right\}$ in the open interval $(0,1)$ such that $t_{n}>\left|z_{n}\right|(n=1,2, \ldots)$ and

$$
\sum_{n \geq 1}\left(1-t_{n}\right)<\infty .
$$

We denote by $C_{n}$ the circle $\partial \Delta\left(0, t_{n}\right)$ and by $p_{n}(z)$ the radial projection of $z$ in $\bar{\Delta}\left(0, t_{n}\right)$ into $C_{n}$, i.e., $p_{n}(z) \in C_{n}$ is characterized by $z \in\left[0, p_{n}(z)\right]$, where we make the convention $p_{n}(0)=t_{n}$ inessential but just for the definiteness. For simplicity we set $p_{n}\left(z_{n}\right)=a_{n}$ and $P_{n}=P \cap \bar{\Delta}\left(0, t_{n}\right)$.

We now construct $\left\{w_{n}\right\}$ inductively. For the choice of $w_{1}$ we consider the set $E_{1}=p_{1}\left(P_{1}\right) \subset C_{1}$, which is a finite subset of $C_{1}$. Therefore we can fix a $w_{1} \in C_{1}$ such that

$$
0<\left|w_{1}-a_{1}\right|<\min \left(\min \left\{\left|c-a_{1}\right|: c \in E_{1} \backslash\left\{a_{1}\right\}\right\}, t_{1}-\left|z_{1}\right|\right),
$$

where $a_{1}=p_{1}\left(z_{1}\right)$. By the choice of $w_{1}$ it is clear that the intersection $\left[z_{1}, w_{1}\right] \cap P=\varnothing$.

In addition to $w_{1}$ suppose we have chosen $w_{j} \in C_{j}$ for each $j=1, \ldots, n-1$ $(n \geq 2)$ by

$$
0<\left|w_{j}-a_{j}\right|<\min \left(\min \left\{\left|c-a_{j}\right|: c \in E_{j} \backslash\left\{a_{j}\right\}\right\}, t_{j}-\left|z_{j}\right|\right),
$$

where $a_{j}=p_{j}\left(z_{j}\right)$ and

$$
E_{j}=p_{j}\left(P_{j} \cup\left\{w_{1}, \ldots, w_{j-1}\right\}\right)
$$

for $j=2, \ldots, n-1$.

Define $E_{n}$ by (5.6) with $j=n$ and recall that $a_{n}=p_{n}\left(z_{n}\right)$. Since $E_{n}$ is a finite subset of $C_{n}$, we can fix a $w_{n}$ in $C_{n}$ satisfying (5.5) with $j=n$. We have thus completed the construction of the sequence $\left\{w_{n}\right\} \quad(n \geq 1)$ in $\Delta$.

We will show that, for any positive integer $n \geq 2$,

$$
\left[z_{n}, w_{n}\right] \cap\left[z_{j}, w_{j}\right]=\varnothing \quad(j=1, \ldots, n-1) .
$$

To see this fix an arbitrary $j=1, \ldots, n-1$. If $\left|z_{n}\right|>t_{j}$, then the above relation (5.7) is trivially true. If $\left|z_{n}\right| \leq t_{j}$, then by the construction of $w_{j}$, we see that $\left[z_{n}, a_{n}\right] \cap\left[z_{j}, w_{j}\right]=\varnothing$. Therefore, again by the construction of $w_{n}$, we have (5.7).

From (5.4) the convergence (5.1) readily follows. Since (5.7) is true for every $n$, we see that (5.2) is valid. By the construction (5.5) of $w_{n}$ and the triangle inequality we have inequalities

$$
\left|z_{n}-w_{n}\right|<2\left(t_{n}-\left|z_{n}\right|\right) \quad(n=1,2, \ldots),
$$

and therefore (5.3) is satisfied. 
5.3. Let $\left\{z_{n}\right\}$ be the determining sequence in $\Delta$ of the given covering surface $\left(\Delta^{\sim}, \Delta, \pi\right)$ and $\left\{w_{n}\right\}$ be the sequence associated with $\left\{z_{n}\right\}$ satisfying (5.1), (5.2), and (5.3). We denote by $\left(\Delta_{1}^{\sim}, \Delta, \pi_{1}\right)$ the two sheeted covering surface whose determining sequence is $\left\{w_{n}\right\}$. Thus $\left(\Delta_{1}^{\sim}, \Delta, \pi_{1}\right)$ is obtained from $\left(\Delta^{\sim}, \Delta, \pi\right)$ by sweeping out of its branch points.

Observe that

$$
\delta_{n}=\left\{z \in \mathbf{C}:\left|z-z_{n}\right|+\left|z-w_{n}\right| \leq s_{n}+\left|z_{n}-w_{n}\right|\right\} \quad\left(s_{n}>0\right)
$$

is a closed ellipse about $z_{n}$ and also $w_{n}$ such that $\delta_{n} \downarrow\left[z_{n}, w_{n}\right]$ as $s_{n} \downarrow 0$. By (5.2) and (5.3) we can inductively choose $s_{n}>0$ so small that $\delta_{n} \quad(n=$ $1,2, \ldots)$ are contained in $\Delta$, mutually disjoint and

$$
\lim _{n \rightarrow \infty} \operatorname{diam} \delta_{n}=0 \text {. }
$$

Thus $\left\{\delta_{n}\right\}$ is an admissible sequence of ellipses in $\Delta$ associated both with $\left(\Delta^{\sim}, \Delta, \pi\right)$ and $\left(\Delta_{1}^{\sim}, \Delta, \pi_{1}\right)$.

We have thus obtained new covering surfaces $\left(d^{\sim}, d, \pi\right)$ and $\left(d_{1}^{\sim}, d, \pi_{1}\right)$ of the subregion $d=\Delta \backslash \bigcup_{k \geq 1} \delta_{k}$ of $\Delta$ associated with $\left(\Delta^{\sim}, \Delta, \pi\right)$ and $\left(\Delta_{1}^{\sim}, \Delta\right.$, $\left.\pi_{1}\right)$, respectively.

5.4. We now show that there exists a conformal mapping $T^{\sim}$ of $d^{\sim}$ onto $d_{1}^{\sim}$ such that

$$
\pi=\pi_{1} \circ T^{\sim}
$$

For the purpose we construct a homeomorphism $T$ of $\Delta$ onto itself such that $T \mid d=$ id (identity) and

$$
T\left(z_{n}\right)=w_{n} \quad(n=1,2, \ldots),
$$

so that $T$ is a suitable topological deformation of $\Delta$ only inside the ellipse $\delta_{n}$ for each $n \geq 1$.

Consider the covering surface $\left(\Delta^{\sim}, \Delta, T \circ \pi\right)$ of $\Delta$. Since its determining sequence is $\left\{w_{n}\right\},\left(\Delta^{\sim}, \Delta, T \circ \pi\right)$ is homeomorphic to $\left(\Delta_{1}^{\sim}, \Delta, \pi_{1}\right)$ as covering surfaces so that there exists a homeomorphism $T^{\sim}$ of $\Delta^{\sim}$ onto $\Delta_{1}^{\sim}$ such that

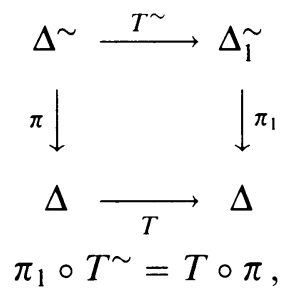

on $\Delta^{\sim}$.

For the sake of completeness we pause here to explain how to construct such a homeomorphism $T^{\sim}$ as described above. Various ways can be considered for the purpose but we use here the so called "construction by scissors and paste" which is the most intuitive one among them. If we denote by $\tau\left(\tau_{1}\right.$, resp.) the cover transformation of $\Delta^{\sim}\left(\Delta_{1}^{\sim}\right.$, resp.), then $\tau_{1}^{n} \circ T^{\sim} \circ \tau^{m}$ for $n=0,1$ and $m=0,1$ are homeomorphisms of $\Delta^{\sim}$ onto $\Delta_{1}^{\sim}$ with (5.9) along with $T^{\sim}$ and actually exhaust all the required ones. Hence we can deduce from the above after a thought that there exist exactly two such homeomorphisms. We will construct a $T^{\sim}$ among these two. 
Since $\Delta \backslash\left\{z_{n}\right\}_{n \geq 1}$ is a region we can find and then fix a simple arc $\gamma_{1}$ in this region connecting $z_{1}$ and $z_{2}$. Observe that $\Delta \backslash \gamma_{1} \cup\left\{z_{n}\right\}_{n \geq 3}$ is also a region so that we can find and then fix a simple arc $\gamma_{2}$ in this region connecting $z_{3}$ and $z_{4}$. Continuing this process we can take and then fix a family $\left\{\gamma_{n}\right\}_{n \geq 1}$ of simple arcs in $\Delta$ with the following two properties: $\gamma_{n}$ connects $z_{2 n-1}$ and $z_{2 n}(n=1,2, \ldots), \gamma_{n} \cap \gamma_{m}=\varnothing(n \neq m)$. Then we consider the slit region

$$
R=\Delta \backslash \bigcup_{n \geq 1} \gamma_{n}
$$

The slit $\gamma_{n}$ gives two sides to $R$ which we denote by $\alpha_{n}$ and $\beta_{n}$ for each $n=1,2, \ldots$. Viewing $\alpha_{n} \cup \beta_{n}$ as a closed curve which forms a border of $R$ for each $n=1,2, \ldots$, we consider the bordered region $\widehat{R}$ obtained from $R$ by attaching borders $\alpha_{n} \cup \beta_{n}(n=1,2, \ldots)$ to $R$. We prepare two copies $\widehat{R}^{+}$and $\widehat{R}^{-}$of $\widehat{R}$ in which $\alpha_{n}^{ \pm}$and $\beta_{n}^{ \pm}$respectively indicate the copies of $\alpha_{n}$ and $\beta_{n}$ in $\widehat{R}^{ \pm}(n=1,2, \ldots)$. Then $\Delta^{\sim}$ is obtained as the union $\widehat{R}^{+} \cup \widehat{R}^{-}$by identifying the points on $\alpha_{n}^{ \pm}$with the corresponding points on $\beta_{n}^{\mp}(n=1,2, \ldots)$. The procedure is usually expressed as pasting $R^{+}$to $R^{-}$crosswise along each $\gamma_{n}$, where $R^{ \pm}$is the interior of $\widehat{R}^{ \pm}$. Here $\pi$ is given as the "natural projection" from $\Delta^{\sim}$ onto $\Delta$.

We repeat entirely the same procedure as above by using $\left\{\boldsymbol{T}\left(\gamma_{n}\right)\right\}_{n \geq 1}$ the family of mutually disjoint simple arcs $T\left(\gamma_{n}\right)$ in $\Delta$ connecting $w_{2 n-1}=T\left(z_{2 n-1}\right)$ and $w_{2 n}=T\left(z_{2 n}\right) \quad(n=1,2, \ldots)$. Namely, we consider the slit region

$$
R_{1}=\Delta \backslash \bigcup_{n \geq 1} T\left(\gamma_{n}\right)
$$

Both sides of the slit $T\left(\gamma_{n}\right)$ may naturally be denoted by $T\left(\alpha_{n}\right)$ and $T\left(\beta_{n}\right)$ for each $n=1,2, \ldots$ so that $T$ gives rise to a homeomorphism, which we also denote by $T$, of the bordered region $\widehat{R}$ onto the bordered region $\widehat{R}_{1}$ obtained from $R_{1}$ by attaching borders $T\left(\alpha_{n}\right) \cup T\left(\beta_{n}\right) \quad(n=1,2, \ldots)$ to $R_{1}$. Preparing two copies $\widehat{R}_{1}^{ \pm}$of $\widehat{R}_{1}$ we construct $\Delta_{1}^{\sim}$ as the union $\widehat{R}_{1}^{+} \cup \widehat{R}_{1}^{-}$by identifying $T\left(\alpha_{n}^{ \pm}\right)$with $T\left(\beta_{n}^{\mp}\right)$ for each $n=1,2, \ldots$. Here again $\pi_{1}$ is obtained as the natural projection of $\Delta_{1}^{\sim}$ onto $\Delta$.

We now define a mapping $T^{\sim}$ from $\Delta^{\sim}$ to $\Delta_{1}^{\sim}$ as follows: first we define $T^{\sim}$ on $R^{ \pm}$as the mapping from $R^{ \pm}$onto $R_{1}^{ \pm}$induced by $T$, i.e., $T^{\sim}(p)=$ $T(p) \in R_{1}^{ \pm}$for $p \in R^{ \pm}$; secondly, since $\alpha_{n}^{ \pm}=\beta_{n}^{\mp}$ in $\Delta^{\sim}$ and $T\left(\alpha_{n}^{ \pm}\right)=T\left(\beta_{n}^{\mp}\right)$ in $\Delta_{1}^{\sim}$ by our construction, we can define

$$
T^{\sim}(p)=T(p) \in T\left(\alpha_{n}^{ \pm}\right)=T\left(\beta_{n}^{\mp}\right) \quad\left(p \in \alpha_{n}^{ \pm}=\beta_{n}^{\mp}\right) .
$$

By the fact that $T$ may be viewed as the homeomorphism of $\widehat{R}^{ \pm}$onto $\widehat{R}_{1}^{ \pm}$and by the construction of $\Delta^{\sim}$ and $\Delta_{1}^{\sim}$ as described above, we can conclude that the mapping $T^{\sim}$ defined as above is a homeomorphism of $\Delta^{\sim}$ onto $\Delta_{1}^{\sim}$ satisfying (5.9). Having finished the construction of a desired $T^{\sim}$ we now return to the main stream of our discussion. 
The restriction of $T^{\sim}$ on $d^{\sim}$, denoted by $T^{\sim}$ again, is then a homeomorphism of $d^{\sim}$ onto $d_{1}^{\sim}$ and we at once deduce (5.8) from (5.9). Since $T^{\sim}=$

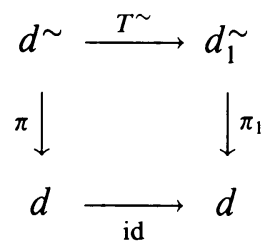

$\pi_{1}^{-1} \circ \pi$ locally at each point of $d^{\sim}$, we see that $T^{\sim}$ is holomorphic at each point of $d^{\sim}$ and a fortiori $T^{\sim}$ is a conformal mapping of $d^{\sim}$ onto $d_{1}^{\sim}$.

5.5. Because of $(5.1)$ and $w_{n} \neq 0 \quad(n \geq 1)$, we can consider on $\Delta$ the Blaschke product

$$
B(z)=\prod_{n \geq 1} \frac{-\bar{w}_{n}}{\left|w_{n}\right|} \cdot \frac{z-w_{n}}{1-\bar{w}_{n} z}
$$

which belongs to $H^{\infty}(\Delta)$. The square root $\sqrt{B(z)}$ defines a single-valued bounded analytic function $\sqrt{B}$ on $\Delta_{1}^{\sim}$ such that

$$
\sqrt{B}\left(z_{1}^{+}\right)=-\sqrt{B}\left(z_{1}^{-}\right) \neq 0,
$$

for every $z$ in $\Delta \backslash\left\{w_{n}\right\}$, where $\pi_{1}^{-1}(z)=\left\{z_{1}^{+}, z_{1}^{-}\right\}$. Hence in particular $\sqrt{B}$ belongs to $H^{\infty}\left(d_{1}^{\sim}\right)$ and (5.10) holds for any $z \in d$.

Now consider the function $f$ in $H^{\infty}\left(d^{\sim}\right)$ defined by $f=\sqrt{B} \circ T^{\sim}$. For any $z \in d$, let $\pi^{-1}(z)=\left\{z^{+}, z^{-}\right\}$. Then, by (5.8), we have $\pi_{1}\left(T^{\sim} z^{ \pm}\right)=\pi\left(z^{ \pm}\right)=$ $z$, and thus $\pi_{1}^{-1}(z)=\left\{T^{\sim} z^{+}, T^{\sim} z^{-}\right\}$. Therefore by (5.10) we see that

$$
f\left(z^{+}\right)=\sqrt{B}\left(T^{\sim} z^{+}\right)=-\sqrt{B}\left(T^{\sim} z^{-}\right)=-f\left(z^{-}\right) \neq 0,
$$

which means that $f\left(z^{+}\right) \neq f\left(z^{-}\right)$. Thus $H^{\infty}\left(d^{\sim}\right)$ separates the points in the fiber $\pi^{-1}(z)$ for any $z$ in $d$ and hence $H^{\infty}\left(d^{\sim}\right)$ separates the points in $d^{\sim}$ since $H^{\infty}\left(d^{\sim}\right) \supset H^{\infty}(d) \circ \pi$.

The proof of Theorem 4 started in 5.2 is herewith complete.

\section{REFERENCES}

1. S. Fisher, Function theory on planar domains, Wiley, 1983.

2. F. Forelli, A note on divisibility in $H^{\infty}(X)$, Canad. J. Math. 38 (1984), 458-469.

3. J. Garnett, Bounded analytic functions, Academic Press, New York, 1981.

4. M. Hayashi and M. Nakai, Point separation by bounded analytic functions of a covering Riemann surface, Pacific J. Math. 134 (1988), 261-273.

5. _- On the Myrberg type phenomenon, Analytic Function Theory of One Complex Variable (C. C. Yang, Y. Komatsu and K. Niino, eds.), Pitman Research Notes in Mathematics Series (Longman Scientific \& Technical), vol. 212, Pitman, New York, 1989, pp. 1-12.

6. P. J. Myrberg, Über die Analytische Fortsetzung von beschränkten Funktionen, Ann. Acad. Sci. Fenn. Ser. A. I. Math. 45 no. 58 (1949), 7 pp.

7. H. L. Royden, Algebras of bounded analytic functions on Riemann surfaces, Acta Math. 114 (1965), 113-142.

8. L. Sario and M. Nakai, Classification theory of Riemann surfaces, Springer-Verlag, 1970.

9. S. Segawa, Bounded analytic functions on unbounded covering surface, Pacific J. Math. 79 (1978), 183-187. 
10. H. L. Selberg, Ein Satz über beschränkte endlichvieldeutige analytische Funktionen, Comment. Math. Helv. 9 (1937), 104-108.

11. C. M. Stanton, Bounded analytic functions on a class of Riemann surfaces, Pacific J. Math. 59 (1975), 557-565.

12. M. Tsuji, Potential theory in modern function theory, Chelsea, 1975.

13. Y. Yamamura, On the existence of bounded analytic functions, Sci. Rep. Tokyo Kyoiku Daigaku Sect. A 10 (1969), 88-102.

Department of Mathematics, Hokkaido University, Sapporo 060, Japan

E-mail address: E12732@sinet.ad.jp

Department of Mathematics, Nagoya Institute of Technology, Gokiso, Showa, NAGOYA 466, JAPAN

Department of Mathematics, Daido Institute of Technology, Daido, Minami, Nagoya 457, JAPAN 